\title{
GROUP-LIKE EXTENSIONS OF MINIMAL SETS
}

\author{
BY \\ ROBERT ELLIS( $\left.{ }^{(}\right)$
}

Introduction. In [1] it was shown that every point transitive transformation group $(X, T)$ with compact Hausdorff phase space $X$ could be represented as the set of $T$-homomorphisms of $\mathscr{A}$ into $\mathscr{C}=\mathscr{C}(\beta T)$, the algebra of bounded functions on $T$, for some $T$-subalgebra $\mathscr{A}$ of $\mathscr{C}(\beta T)$. In this context it seems natural to try to obtain information about $(X, T)$ by means of algebraic objects naturally attached to $\mathscr{A}$. One such is a group $G(\mathscr{A})$ of $T$-homomorphisms of $\mathscr{C}$ into $\mathscr{C}$ which leave the elements of $\mathscr{A}$ elementwise fixed.

Let $(X, T)$ and $(Y, T)$ be minimal sets with compact Hausdorff phase spaces, let $(Y, T)$ be a homomorphic image of $(X, T)$, and let $\mathscr{A}, \mathscr{B}$ be algebras corresponding to $(X, T)$ and $(Y, T)$ respectively. Then we may assume $\mathscr{B} \subset \mathscr{A}$, whence $G(\mathscr{A}) \subset G(\mathscr{B})$. Even in this situation it may happen that $\mathscr{B} \neq \mathscr{A}$ and $G(\mathscr{A})=G(\mathscr{B})$. In order to prevent this one must place some further restrictions on the pair $[(X, T),(Y, T)]$. This leads to the notion of a group-like extension (see 7).

When $Y$ is a one-point space, then $(X, T)$ is a group-like extension of $(Y, T)$ if and only if $(X, T)$ is distal (20). This leads to some results in [6].

Let $(X, T)$ be a group-like extension of $(Y, T)$. Then it may happen that $G(\mathscr{A})$ is a normal subgroup of $G(\mathscr{B})$. When this occurs, $(X, T)$ is called a group extension of $(Y, T)$. The reason for this nomenclature is that $(X, T)$ is a group extension of $(Y, T)$ if and only if there exists a group of homeomorphisms $H$ of $X$ onto $X$ such that $(H, X, T)$ is a bitransformation group [3] with $(X / H, T)$ isomorphic to $(Y, T)$.

When $(X, T)$ is merely a group-like extension of $(Y, T)$ one can show (29) that there exists a group extension $(Z, T)$ of both $(Y, T)$ and $(X, T)$. Let $H$ act on $Z$ with $(Z / H, T)$ isomorphic to $(Y, T)$ and let $F \subset X$ be a fiber over some point of $Y$. Then there exists an action of $H$ on $F$ such that $((Z \times F) / H, T)$ is the homomorphic image of $(X, T)$ under a homomorphism $\phi$ which is also one-one. Thus $(X, T)$ is "almost" the associated fiber space to the "principal" fiber space $(Z, Y)$ with fiber $F$. I write "almost" because in general $\phi$ is not a homeomorphism. The case when $\phi$ is indeed a homeomorphism is a generalization of Furstenberg's theory of isometric extensions [5].

Another natural algebraic approach is to start with a subgroup $H$ of a group $G$ of automorphisms of some universal minimal set and to form $\mathscr{A}(H)$, the subset of $\mathscr{C}(\beta T)$ consisting of those functions left fixed by all the elements of $H$. Then $\mathscr{A}(H)$ is a $T$-subalgebra of $\mathscr{C}(\beta T)$ which corresponds to a minimal set. Now

Received by the editors May 19, 1966.

(1) This research was supported by NSF Grant GP-3580. 
$H \subset G(\mathscr{A}(H))$ and the question arises, when does $H=G(\mathscr{A}(H))$. This leads to the introduction of the $\tau$-topology on $G$ and the solution: $H=G(\mathscr{A}(H))$ if and only if $H$ is a $\tau$-closed subgroup of $G$.

In this paper group-like extensions are studied and the above characterizations proved. The $\tau$-topology is defined on $G$ and developed only so far as is necessary to characterize the closed subgroups. In a subsequent paper a deeper study of this topology will be made and a generalization of Furstenberg's theory obtained therefrom.

Notation. $T$ will denote a discrete group, $\beta T$ its $\beta$-compactification, and $\mathscr{C}$ the algebra of continuous real valued functions on $\beta T$. For $f \in \mathscr{C}, x \in \beta T,\langle f, x\rangle$ will denote the image of $x$ under $f$. Let $f \in \mathscr{C}, u, w \in \beta T$. Then $f u(w f)$ is that element of $\mathscr{C}$ such that $\langle f u, x\rangle=\langle f, u x\rangle(\langle w f, x\rangle=\langle f, x w\rangle)$ for $x \in \beta T$. (Recall that $\beta T$ has a natural semigroup structure making left multiplication continuous [1].)

Let $\mathscr{A}$ be a subalgebra of $\mathscr{C}$. Then $\mathscr{A}$ is a $T$-subalgebra if $\mathscr{A}$ is uniformly closed and $t f \in \mathscr{A},(f \in \mathscr{A}, t \in T)$. ( $T$ may be considered a subset of $\beta T$ so that $t f$ is well defined.) Let $\mathscr{A}$ be a $T$-subalgebra of $\mathscr{C}$. Then an algebra homomorphism $\phi$ of $\mathscr{A}$ into $\mathscr{C}$ is a $T$-homomorphism if $\phi(t f)=t \phi(f)(f \in \mathscr{A}, t \in T)$. The set of $T$-homomorphisms of $\mathscr{A}$ into $\mathscr{C}$ will be denoted $|\mathscr{A}|$.

I shall make constant reference to [1]. However, the following points perhaps should be made explicit.

1. Let $(X, T)$ be a point transitive transformation group with compact phase space $X$. Then there exists a $T$-subalgebra $\mathscr{A}$ of $\mathscr{C}$ and a natural action of $T$ on $|\mathscr{A}|$ such that $(X, T)$ is isomorphic to $(|\mathscr{A}|, T)$.

2. Let $\mathscr{A}$ be a $T$-subalgebra of $\mathscr{C}, \phi \in|\mathscr{A}|$. Then there exists $u \in \beta T$ such that $\phi(f)=f u(f \in \mathscr{A}) ; u$ of course is in general not unique.

Let $\mathscr{A}$ be a $T$-subalgebra of $\mathscr{C}$. Then I will say that $\mathscr{A}$ possesses some recursive property if the corresponding transformation group $(|\mathscr{A}|, T)$ possesses the property in question. Thus $\mathscr{A}$ is minimal if $(|\mathscr{A}|, T)$ is minimal, etc.

My first task will be to determine the universal minimal $T$-subalgebras of $\mathscr{C}$.

For the remainder of the paper $M$ will denote a fixed minimal subset of $(\beta T, T)$ and $J$ the set of idempotents of $M$. (Recall that $M$ is universal.)

1. Proposition. Let $x \in \beta T, \mathscr{A}(x)=[f \mid f x=f]$.

Then 1. $\mathscr{A}(x)$ is a T-subalgebra of $\mathscr{C}$.

2. If $x \in J$ then $\mathscr{A}(x)$ is a universal minimal T-subalgebra of $\mathscr{C}$.

Proof. 1. Verify directly.

2. Let $x \in J$. Then the mapping $L_{x}: \beta T \rightarrow M$ such that $L_{x}(y)=x y(y \in \beta T)$ is a homomorphism of $\beta T$ onto $M$. The image of the induced $T$-homomorphism of $\mathscr{C}(M)$ into $\mathscr{C}$ is just $\mathscr{A}(x)$. The proof is completed.

2. Lemma. Let $u \in J, x \in \beta T$. Then there exists $v \in J$ such that $\mathscr{A}(v)=\mathscr{A}(u) x$.

Proof. There exists $v \in J$ with $u x v=u x$ [2]. Let $f \in \mathscr{A}(u)$. Then $f x v=f u x v$ $=f u x=f x$, whence $f x \in \mathscr{A}(v)$. 
Now let $g \in \mathscr{A}(v)$. There exists $y \in M$ with $y u x=v$. Then $g y u=(g y u) u \in \mathscr{A}(u)$ and $g y u x=g v=g$.

3. Proposition. Let $\mathscr{A}$ be a universal minimal T-subalgebra of $\mathscr{C}$. Then there exists $v \in J$ such that $\mathscr{A}=\mathscr{A}(v)$.

Proof. Since $(M, T)$ and $(|\mathscr{A}|, T)$ are universal minimal sets, they are isomorphic. This isomorphism yields a $T$-isomorphism $\phi$ of $\mathscr{A}(u)$ onto $\mathscr{A}$. There exists $x \in \beta T$ such that $\phi(f)=f x(f \in \mathscr{A}(u))$. Hence $\mathscr{A}=\mathscr{A}(u) x=\mathscr{A}(v)$ for some $v \in J$.

4. Corollary. Let $\mathscr{A}$ be a T-subalgebra of $\mathscr{C}$. Then $\mathscr{A}$ is minimal if and only if $\mathscr{A} \subset \mathscr{A}(u)$ for some $u \in J$.

Proof. If $\mathscr{A} \subset \mathscr{A}(u)$ with $u \in J$, then $|\mathscr{A}|$ is the homomorphic image of $|\mathscr{A}(u)|$. Then $|\mathscr{A}|$ is minimal [4].

Now suppose $\mathscr{A}$ is minimal. Then $|\mathscr{A}|$ is the homomorphic image of $|\mathscr{A}(u)|$. Hence there exists $x \in \beta T$ with $\mathscr{A} x \subset \mathscr{A}(u)$. The mapping $f \rightarrow f x(f \in \mathscr{A})$ of $\mathscr{A}$ onto $\mathscr{A} x$ is a surjective homomorphism. Since $\mathscr{A}$ is minimal it must be injective [1]. Hence there exists $y \in \beta T$ such that $\mathscr{A} x y=\mathscr{A}$. Then $\mathscr{A}=\mathscr{A} x y \subset \mathscr{A}(u) y=\mathscr{A}(v)$ for some $v \in J$.

5. Lemma. Let $\mathscr{A}, \mathscr{B}$ be minimal $T$-subalgebras of $\mathscr{C}$ with $\mathscr{A} \varsubsetneqq \mathscr{B}$. Then there exist $p, y \in M$ with $\mathscr{A} p \subset \mathscr{A}(y)$ and $\mathscr{B} p \notin \mathscr{A}(y)$.

Proof. By Corollary 4 there exists $u \in J$ with $\mathscr{B} \subset \mathscr{A}(u)$. Since $\mathscr{A} \neq \mathscr{B},|\mathscr{A}| \neq|\mathscr{B}|$ and therefore there exist $p, q \in \beta T$ such that $f p=f q(f \in \mathscr{A})$ and $h p \neq h q$ for some $h \in \mathscr{B}$. Since $u p=p$ and $u q=q$ on $\mathscr{B}$, we may assume that $p, q \in M=u(\beta T)$.

Let $y \in M$ with $p y=q$. Then $f p y=f q=f p(f \in \mathscr{A})$ but $h p y=h q \neq h p$. Thus $\mathscr{A} p \subset \mathscr{A}(y)$ but $\mathscr{B} p \notin \mathscr{A}(y)$.

6. Proposition. Let $\mathscr{A}, \mathscr{B}$ be minimal T-subalgebras of $\mathscr{C}$ with $\mathscr{A} \varsubsetneqq \mathscr{B}$. Then either (i) there exists $q \in M$ with $q \mid \mathscr{A}=$ identity and $q \mid \mathscr{B} \neq$ identity, or (ii) there exist $x \in M, w \in J$ with $\mathscr{A} x \subset \mathscr{A}(w)$ and $\mathscr{B} x \notin \mathscr{A}(w)$.

Proof. Assume that (ii) does not hold, i.e. $(x \in M, w \in J) \mathscr{A} x \subset \mathscr{A}(w)$ implies $\mathscr{B} x \subset \mathscr{A}(w)$.

Let $p, y \in M$ be as in Lemma 5, i.e. $\mathscr{A} p \subset \mathscr{A}(y) \ngtr \mathscr{B} p$, and let $v \in J$ with $y v=y$. Then $\mathscr{A} p \subset \mathscr{A}(y) \subset \mathscr{A}(v)$ implies that $\mathscr{B} p \subset \mathscr{A}(v)$. Thus we may assume $p v=p$.

Now let $u \in J$ with $\mathscr{B} \subset \mathscr{A}(u)$. Set $q=p r$ where $p y r=u$. Then $f q=f p r=f p y r$ $=f u=f(f \in \mathscr{A})$; i.e. $q \mid \mathscr{A}=$ identity.

Let $h \in \mathscr{B}$ with $h p \neq h p y$. I claim that $h q \neq h$. To see this let $s \in M$ with $r s=v$ and suppose $h q=h$. Then $h=h u=h p y r$, whence $h s=h p y r s=h p y v=h p y$. On the other hand $h s=h q s=h p r s=h p v=h p$. Thus the supposition $h q=h$ leads to the contradiction $h p=h p y$.

Let $u \in J, \mathscr{A}$ a minimal $T$-subalgebra of $\mathscr{C}$. Then by Corollary 4 there exists $w \in J$ with $\mathscr{A} \subset \mathscr{A}(w)$. This implies that $\mathscr{A} u \subset \mathscr{A}(w) u=\mathscr{A}(u)$. Moreover, since $\mathscr{A}$ is minimal, $\mathscr{A}$ is isomorphic to $\mathscr{A} u$. Thus from the point of view of isomorphism 
types of minimal $T$-subalgebras we may suppose that they are all contained in a fixed universal algebra $\mathscr{M}=\mathscr{A}(u)$, where $u$ is a fixed element of $J$.

However, Proposition 6 indicates that in order to distinguish between two such algebras $\mathscr{A}$ and $\mathscr{B}$ with $\mathscr{A} \subset \mathscr{B}$ we may have to go "outside" of $\mathscr{M}$ and see how the various isomorphic copies of $\mathscr{A}$ and $\mathscr{B}$ are situated with respect to the other universal algebras $\mathscr{A}(w) \mid w \in J)$. In order to distinguish between $\mathscr{A}$ and $\mathscr{B}$ having reference only to $\mathscr{M}$ we must therefore assume that the isomorphic copies of $\mathscr{A}$ and $\mathscr{B}$ are "nicely placed" with respect to the family $(\mathscr{A}(w) \mid w \in J)$. This is the reason for the following definition.

7. Definition. Let $\mathscr{A}, \mathscr{B}$ be minimal $T$-subalgebras of $\mathscr{C}$ with $\mathscr{A} \subset \mathscr{B}$. Then $\mathscr{B}$ is a group-like extension of $\mathscr{A}$ (denoted $\mathscr{B}>\mathscr{A})$ if $(x \in M, w \in J) \mathscr{A} x \subset \mathscr{A}(w)$ implies $\mathscr{B} x \subset \mathscr{A}(w)$; i.e. condition (ii) of Proposition 6 does not hold.

8. Notation. For the remainder of the paper $u$ will denote a fixed element of $J$, $\mathscr{M}$ the universal minimal $T$-subalgebra $\mathscr{A}(u)$, and $G=[p \mid p \in M$ and $p u=p]$. Then $G$ is actually a subgroup of $M$ [2]. To emphasize this fact I shall often denote the elements of $G$ with lower case Greek letters; $\omega$ will denote the identity of $G$. (Note $\omega=u$.)

9. Proposition. Let $\mathscr{A}, \mathscr{B}, \mathscr{D}$ be T-subalgebras of $\mathscr{M}$ with $\mathscr{A} \subset \mathscr{B} \subset \mathscr{D}$. Then (i) $\mathscr{B}>\mathscr{A}$ implies $\mathscr{B} p>\mathscr{A} p(p \in \beta T)$, (ii) $\mathscr{D}>\mathscr{A}$ if and only if $\mathscr{D}>\mathscr{B}$ and $\mathscr{B}>\mathscr{A}$.

Proof. (i) Let $x \in M, w \in J$ and $\mathscr{A} p x \subset \mathscr{A}(w)$. We must show that $\mathscr{B} p x \subset \mathscr{A}(w)$. Now upx $\in M, \mathscr{B}>\mathscr{A}$, and $\mathscr{A}(w) \supset \mathscr{A} p x=\mathscr{A}$ upx imply that $\mathscr{A}(w) \supset \mathscr{B}$ upx $=\mathscr{B} p x$.

(ii) Let $\mathscr{D}>\mathscr{A}$. To show $\mathscr{D}>\mathscr{B}$ and $\mathscr{B}>\mathscr{A}$, let $p, q \in M, v, w \in J$ with $\mathscr{A} p \subset \mathscr{A}(v)$ and $\mathscr{B} q \subset \mathscr{A}(w)$. Then $\mathscr{D}>\mathscr{A}$ implies that $\mathscr{D} p \subset \mathscr{A}(v)$ whence $\mathscr{B} p \subset \mathscr{A}(v)$ since $\mathscr{B} \subset \mathscr{D}$. Thus $\mathscr{B}>\mathscr{A}$. Moreover $\mathscr{A} \subset \mathscr{B}$ and $\mathscr{B} q \subset \mathscr{A}(w)$ imply that $\mathscr{A} q \subset \mathscr{A}(w)$. Hence $\mathscr{D} q \subset \mathscr{A}(w)$. Thus $\mathscr{D}>\mathscr{B}$.

Now suppose $\mathscr{D}>\mathscr{B}$ and $\mathscr{B}>\mathscr{A}$. Let $p \in M, w \in J$ with $\mathscr{A} p \subset \mathscr{A}(w)$. Then $\mathscr{B}>\mathscr{A}$ implies that $\mathscr{B} p \subset \mathscr{A}(w)$ whence $\mathscr{D} p \subset \mathscr{A}(w)$ because $\mathscr{D}>\mathscr{B}$. The proof is completed.

10. Definition. Let $\mathscr{A}$ be a $T$-subalgebra of $\mathscr{M}$. Then $G(\mathscr{A})=[\alpha \mid \alpha \in G, f \alpha=f$, $(f \in \mathscr{A})$ ]. Then it is immediate that $G(\mathscr{A})$ is a subgroup of $G$.

11. Proposition. Let $\mathscr{A}, \mathscr{D}$ be $T$-subalgebras of $\mathscr{M}$ with $\mathscr{D}>\mathscr{A}$, let $\mathscr{E}, \mathscr{F}$ be $T$-subalgebras of $\mathscr{M}$ with $\mathscr{A} \subset \mathscr{F}$ and $\mathscr{E} \cup \mathscr{F} \subset \mathscr{D}$. Then $\mathscr{E} \subset \mathscr{F}$ if and only if $G(\mathscr{F})$ $\subset G(\mathscr{E})$.

Proof. Clearly $\mathscr{E} \subset \mathscr{F}$ implies that $G(\mathscr{F}) \subset G(\mathscr{E})$.

Let $G(\mathscr{F}) \subset G(\mathscr{E})$. Let $\mathscr{L}$ be the $T$-subalgebra of $\mathscr{C}$ generated by $\mathscr{E} \cup \mathscr{F}$. Then $\mathscr{L} \subset \mathscr{D}$ and Proposition 9 implies that $\mathscr{L}>\mathscr{F}$. If $\mathscr{F}$ were not equal to $\mathscr{L}$ there would exist $q \in M$ with $q \mid \mathscr{F}$ equal to the identity and $q \mid \mathscr{L}$ unequal to the identity by 6 and 7. Since $\mathscr{F} \subset \mathscr{L} \subset \mathscr{M}, q=q u$ on $\mathscr{L}$. Hence there would exist $\alpha \in G(\mathscr{F})$ with $\alpha \notin G(\mathscr{L})$. This however is impossible because we have assumed $G(\mathscr{F}) \subset G(\mathscr{E})$; indeed $\alpha \in G(\mathscr{F})$ implies $\alpha \in G(\mathscr{E})$ whence $\alpha \in G(\mathscr{L})$ by definition of $\mathscr{L}$. 
12. Definition. Let $A \subset \beta T, f \in \mathscr{C}$. Then $f_{A}$ will denote that element of $\mathscr{C}$ given by $\left\langle f_{A}, t\right\rangle=\sup [\langle f a, t\rangle \mid a \in A](t \in T)$.

Let $K \subset G$. Then the $\tau$-closure of $K$ (denoted $\operatorname{cls}_{\tau} K$ ) is the set $\left[\alpha \mid \alpha \in G, f \alpha \leqq f_{K} \omega\right.$ $(f \in \mathscr{M})$ ]. The topology (see Proposition 16) defined by this closure operator will be referred to as the $\tau$-topology on $G$.

13. Lemma. Let $K \subset G$. Then $(t f)_{K}=t\left(f_{K}\right)(t \in T, f \in \mathscr{M})$.

Proof. Let $f \in \mathscr{M}, t, s \in T$. Then $\langle t f \alpha, s\rangle=\langle f \alpha, s t\rangle \leqq\left\langle f_{K}, s t\right\rangle=\left\langle t f_{K}, s\right\rangle(\alpha \in K)$. Hence $(t f)_{K} \leqq t f_{K}$.

Also $\langle t f \alpha, s\rangle \leqq\left\langle(t f)_{K}, s\right\rangle(\alpha \in K)$ implies that $\langle f \alpha, s t\rangle \leqq\left\langle t^{-1}(t f)_{K}, s t\right\rangle$ whence $f_{K} \leqq t^{-1}(t f)_{K}$ and $t f_{K} \leqq(t f)_{K}$.

14. Lemma. Let $L, K \subset G$. Then (i) $K \subset \operatorname{cls}_{\tau} K$, (ii) $L \subset K$ implies $\operatorname{cls}_{\tau} L \subset \operatorname{cls}_{\imath} K$, (iii) $\operatorname{cls}_{\tau} K=\operatorname{cls}_{\imath}\left(\operatorname{cls}_{\tau} K\right)$.

Proof. (i) and (ii) are immediate.

(iii) $K \subset \mathrm{cls}_{\imath} K$ by (i). Hence $\operatorname{cls}_{\imath} K \subset \operatorname{cls}_{\imath}\left(\operatorname{cls}_{\imath} K\right)$ by (ii).

Set $N=\operatorname{cls}_{\imath} K$. Then $\langle f \alpha, t\rangle \leqq\left\langle f_{K} \omega, t\right\rangle(\alpha \in N, t \in T, f \in \mathscr{M})$. Hence $\left\langle f_{N}, t\right\rangle$ $\leqq\left\langle f_{K} \omega, t\right\rangle(t \in T, f \in \mathscr{M})$. Thus $f_{N} \leqq f_{K} \omega$, whence $f_{N} \omega \leqq f_{K} \omega^{2}=f_{K} \omega$.

Let $\alpha \in \operatorname{cls}_{\tau} N$. Then $f \alpha \leqq f_{N} \omega=f_{K} \omega(f \in \mathscr{M})$. Hence $\alpha \in \operatorname{cls}_{\imath} K$.

15. Lemma. Let $K \subset G$. Then $K=\operatorname{cls}_{\tau} K$ if and only if given $\alpha \notin K$ there exists $f \in \mathscr{M}$ such that $\left\langle f_{K}, \omega\right\rangle\langle\langle f, \alpha\rangle$.

Proof. Let $K=\operatorname{cls}_{\tau} K$ and suppose $\alpha \notin K$. Then there exists $g \in \mathscr{M}$ with $\left.g \alpha\right\rfloor g_{K} \omega$. Hence there exists $t \in T$ such that $\langle t g, \alpha\rangle=\langle g, \alpha t\rangle=\langle g \alpha, t\rangle\rangle\left\langle g_{K} \omega, t\right\rangle=\left\langle\operatorname{tg}_{K}, \omega\right\rangle$ $=\left\langle(t g)_{K}, \omega\right\rangle$. Set $f=t g$. Then $f \in \mathscr{M}$ and $\left\langle f_{K}, \omega\right\rangle\langle\langle f, \alpha\rangle$.

Now suppose the condition of Lemma 15 to be satisfied. If $\alpha \notin K$ then $\left\langle f_{K}, \omega\right\rangle$ $\langle\langle f, \alpha\rangle$ for some $f \in \mathscr{M}$. Hence $f \alpha\rfloor f_{K} \omega$ and $\alpha \notin \operatorname{cls}_{\tau} K$.

16. Proposition. The map $K \rightarrow \mathrm{cls}_{\imath} K$ of $\mathscr{P} G$ into $\mathscr{P} G$ is a closure operator on $G$.

Proof. By 14 it suffices to show $\operatorname{cls}_{\imath}\left(K_{1} \cup K_{2}\right) \subset \operatorname{cls}_{\imath} K_{1} \cup \operatorname{cls}_{\tau} K_{2}$ for two nonnull subsets $K_{1}$ and $K_{2}$ of $G$.

Let $\alpha \in G$ with $\alpha \notin \operatorname{cls}_{\tau} K_{1} \cup \operatorname{cls}_{\tau} K_{2}$. By 15 there exist $f^{1}, f^{2} \in \mathscr{M}$ such that $\left\langle f_{K_{i}}^{i}, \omega\right\rangle\left\langle\left\langle f^{i}, \alpha\right\rangle(i=1,2)\right.$. By adding constant functions to the $f^{i}$ we may assume that $\left\langle f^{1}, \alpha\right\rangle=\left\langle f^{2}, \alpha\right\rangle$. Since all the functions involved are continuous there exist a neighborhood $N$ of $\omega$ and a real number $b$ such that $\left\langle f_{K_{1}}^{i}, t\right\rangle\left\langle b\left\langle\left\langle f^{i}, \alpha\right\rangle\right.\right.$ $(t \in N, i=1,2)$. Set $\langle g, p\rangle=\inf \left[\left\langle f^{i}, p\right\rangle \mid i=1,2\right](p \in \beta T)$. Then $g \in \mathscr{M}$ since $\mathscr{M}$ is a subalgebra of $\mathscr{C}$. Let $\beta \in K_{1} \cup K_{2}$. Then $\beta \in K_{i}$ for $i=1$ or 2 . Hence $\langle g \beta, t\rangle$ $=\langle g, \beta t\rangle \leqq\left\langle f^{i}, \beta t\right\rangle=\left\langle f^{i} \beta, t\right\rangle \leqq\left\langle f_{K_{i}}^{i}, t\right\rangle\left\langle b\left\langle\left\langle f^{i}, \alpha\right\rangle=\langle g, \alpha\rangle(t \in N)\right.\right.$. Thus

$$
\left\langle g_{K_{1} \cup K_{2}}, t\right\rangle \leqq b<\langle g, \alpha\rangle \quad(t \in N) .
$$

Since $g_{K_{1} \cup K_{2}}$ is continuous and $T$ is dense in $\beta T,\left\langle g_{K_{1} \cup K_{2}}, \omega\right\rangle\langle\langle g, \alpha\rangle$; i.e. $\left\langle g_{K_{1} \cup K_{2}} \omega, e\right\rangle\left\langle\langle g \alpha, e\rangle\right.$. Hence $\alpha \notin \operatorname{cls}_{\imath} K_{1} \cup K_{2}$. The proof is completed. (It will 
be shown in a subsequent paper that $(G, \tau)$ is compact $T_{1}$, that inversion is continuous, and that multiplication is unilaterally continuous.)

17. LemMA. Let $\mathscr{A}$ be a T-subalgebra of $\mathscr{M}$. Then $G(\mathscr{A})$ is a closed subgroup of $(G, \tau)$.

Proof. Let $\alpha \notin G(\mathscr{A})$. Then there exists $f \in \mathscr{A}$ with $f \alpha \neq f$. Hence there exists $t \in T$ with $\langle f, t\rangle \neq\langle f \alpha, t\rangle$, or $\langle t f, e\rangle \neq\langle t f, \alpha\rangle$. Since $(-f) \in \mathscr{A}$, we may assume $\langle t f, e\rangle\langle\langle t f, \alpha\rangle$. Set $g=t f \in \mathscr{A}$. Then $g \omega=g$ implies that $\langle g, \omega\rangle\langle\langle g, \alpha\rangle$. Moreover, $g \beta=g(\beta \in G(\mathscr{A}))$ implies that $g_{G(\mathscr{A})}=g$. Hence $\left\langle g_{G(\mathscr{A})}, \omega\right\rangle\langle\langle g, \alpha\rangle$. The proof is completed.

18. Proposition. Let $H$ be a closed subgroup of $(G, \tau), \mathscr{A}(H)=[f \mid f \in \mathscr{M}$, $f \alpha=f(\alpha \in H)$ ]. Then $\mathscr{A}(H)$ is a T-subalgebra of $\mathscr{M}$ and $G(\mathscr{A}(H))=H$.

Proof. That $\mathscr{A}(H)$ is a $T$-subalgebra of $\mathscr{M}$ is immediate. It is also clear that $H \subset G(\mathscr{A}(H))$.

Now let $\alpha \notin H$. Then there exists $f \in \mathscr{M}$ with $\left\langle f_{H}, \omega\right\rangle\langle\langle f, \alpha\rangle$.

Let $\beta \in H, t \in T$. Then $\left\langle f_{H} \beta, t\right\rangle=\left\langle f_{H}, \beta t\right\rangle \geqq\langle f \gamma, \beta t\rangle(\gamma \in H)$. Set $\gamma=\delta \beta^{-1}$. Then $\left\langle f_{H} \beta, t\right\rangle \geqq\langle f \delta, t\rangle(\delta \in H)$. Consequently $f_{H} \beta \geqq f_{H}(\beta \in H)$.

Set $g=f_{H} \omega$. Then $g=g \omega$ implies that $g \in \mathscr{M}$. Also $g \beta=f_{H} \beta \geqq f_{H}(\beta \in H)$; whence $g \beta=g \beta \omega \geqq f_{H} \omega=g(\beta \in H)$. Thus $g \beta=g(\beta \in H)$. Therefore $g \in \mathscr{A}(H)$. But $\langle g, e\rangle$ $=\left\langle f_{H} \omega, e\right\rangle=\left\langle f_{H}, \omega\right\rangle\left\langle\langle f, \alpha\rangle=\langle f \omega, \alpha\rangle \leqq\left\langle f_{H}, \alpha\right\rangle=\left\langle f_{H}, \omega \alpha\right\rangle=\left\langle f_{H} \omega, \alpha\right\rangle=\langle g, \alpha\rangle\right.$; whence $g \neq g \alpha$. Thus $\alpha \notin G(\mathscr{A}(H))$.

Proposition 18 shows that the mapping $H \rightarrow \mathscr{A}(H)$ is injective on closed subgroups of $G$ and Proposition 11 shows that the mapping $\mathscr{B} \rightarrow G(\mathscr{B})$ is also injective when its domain is properly restricted. The above maps are indeed inverse to one another when properly restricted. This restriction is necessitated because of the limitations of Proposition 11.

19. Proposition. Let $\mathscr{A}$ and $\mathscr{D}$ be T-subalgebras of $\mathscr{M}$ with $\mathscr{D}>\mathscr{A}$ and $\mathscr{D}=\mathscr{A}(G(\mathscr{D}))$. Then there is a one-one correspondence between $T$-subalgebras $\mathscr{B}$ with $\mathscr{A} \subset \mathscr{B} \subset \mathscr{D}$ and closed subgroups $H$ of $(G, \tau)$ with $G(\mathscr{D}) \subset H \subset G(\mathscr{A})$. The correspondence is given by $\mathscr{B} \rightarrow G(\mathscr{B})$, the inverse mapping being $H \rightarrow \mathscr{A}(H)$.

Proof. Let $\mathscr{B}$ be a $T$-subalgebra with $\mathscr{A} \subset \mathscr{B} \subset \mathscr{D}$. Then $G(\mathscr{D}) \subset G(\mathscr{B})$ implies that $\mathscr{B} \subset \mathscr{A}(G(\mathscr{B})) \subset \mathscr{A}(G(\mathscr{D}))=\mathscr{D}$. By Proposition $18 G(\mathscr{A}(G(\mathscr{B})))=G(\mathscr{B})$. Hence $\mathscr{B}=\mathscr{A}(G(\mathscr{B}))$ by Proposition 11 . The proof is completed.

I would now like to investigate the topological significance of group-like extensions. First I shall consider the case of group-like extensions of the algebra $R$ of constant functions. The transformation group $|R|$ is of course the trivial one.

19. Lemma. Let $\mathscr{A}$ be a $T$-subalgebra of $\mathscr{M}$. Then $\mathscr{A}>R$ if and only if $f p=f p v$ $(f \in \mathscr{A}, v \in J, p \in M)$.

Proof. Let $\mathscr{A}>R, f \in \mathscr{A}, v \in J, p \in M$. Since $R p=R \subset \mathscr{A}(v), \mathscr{A} p \subset \mathscr{A}(v)$; whence $f p=f p v$. 
Conversely, assume $f p v=f p(f \in \mathscr{A}, v \in J, p \in M)$. Then $\mathscr{A} p \subset \mathscr{A}(v)(p \in M$, $v \in J$ ) whence $\mathscr{A}>R$.

20. Proposition. Let $\mathscr{A}$ be a T-subalgebra of $\mathscr{M}$. Then $\mathscr{A}>R$ if and only if $\mathscr{A}$ is distal.

Proof. Let $\mathscr{D}$ be the $T$-subalgebra of $\mathscr{C}$ generated by $[\mathscr{A} p \mid p \in M]$. Then $|\mathscr{D}|$ is the enveloping semigroup of $|\mathscr{A}|[1]$ and $\mathscr{A}$ is distal if and only if $\mathscr{D}$ is minimal [2].

Assume that $\mathscr{A}$ is distal, let $f \in \mathscr{A}, v \in J, p \in M$. Then $\mathscr{D}$ is minimal. Hence there exists $w \in J$ with $\mathscr{D} \subset \mathscr{A}(w)$ by 4 . Then $f p v w=f p v$ since $f p v \in \mathscr{D}$. But $v w=w$ implies that $f p v=f p w$. Moreover, $f p \in \mathscr{D}$. Hence $f p=f p w$. Therefore $f p v=f p$, whence $\mathscr{A}>R$ by 19 .

Now assume $\mathscr{A}>R$. Then by $19 \mathscr{A} p \subset \mathscr{A}(u)=\mathscr{M}(p \in M)$. Hence $\mathscr{D} \subset \mathscr{M}$. Therefore $\mathscr{D}$ is minimal whence $\mathscr{A}$ is distal.

21. Proposition. Let $\mathscr{A}, \mathscr{B}$ be $T$-subalgebras of $\mathscr{M}$, let $\mathscr{A}$ be distal and $\mathscr{D}$ the $T$-subalgebra generated by $\mathscr{A} \cup \mathscr{B}$. Then $\mathscr{D}>\mathscr{B}$.

Proof. Let $p \in M, w \in J$ with $\mathscr{B} p \subset \mathscr{A}(w)$. Set $\mathscr{E}=[f \mid f \in \mathscr{D}, f p \in \mathscr{A}(w)]$. Then $\mathscr{E}$ is a uniformly closed $T$-subalgebra of $\mathscr{D}$ which contains $\mathscr{B}$. It also contains $\mathscr{A}$ by 19 and 20. Hence $\mathscr{D}=\mathscr{E}$; i.e. $\mathscr{D} p \subset \mathscr{A}(w)$. The proof is completed.

22. Remarks. 1. Combining 19 and 20 one obtains that $\mathscr{A}$ is distal if and only if $f p v=f p(f \in \mathscr{A}, p \in M, v \in J)$. This is the characterization of distal algebras given in [6].

2. Proposition 21 gives a method of obtaining group-like extensions of a given minimal algebra; just add distal functions to it.

3. Propositions 20 and 21 also show that the collection of distal functions forms an algebra.

In [3] the notion of a bitransformation group was introduced; i.e. a triple $(H, X, T)$ where $(H, X)$ and $(X, T)$ are transformation groups with phase space $X$ and phase groups $H$ and $T$ respectively such that $(h x) t=h(x t)(h \in H, x \in X$, $t \in T)$. In this case $T$ acts on the orbit space $X / H=[H x \mid x \in X]$ in a natural manner. It will be shown that the transformation group $(Y, T)$ with $(Y, T)$ minimal and $Y$ compact $T_{2}$, is of the form $(X / H, T)$, for some bitransformation group $(H, X, T)$ with $(X, T)$ minimal and $X$ compact $T_{2}$, if and only if $\mathscr{B}>\mathscr{A}$ and $G(\mathscr{B})$ is a normal subgroup of $G(\mathscr{A})$, where $\mathscr{B}$ corresponds to $X$ and $\mathscr{A}$ to $Y$.

23. LEMMA. Let $\mathscr{A}, \mathscr{B}$ be $T$-subalgebras of $\mathscr{M}$ with $\mathscr{B}>\mathscr{A}$, let $p, q \in M$ with $p|\mathscr{A}=q| \mathscr{A}$. Then there exists $\alpha \in G(\mathscr{A})$ with $\alpha p=q$ on $\mathscr{B}$. If, moreover, $p|\mathscr{B}=q| \mathscr{B}$, then $\alpha \in G(\mathscr{B})$.

Proof. Let $v \in J$ with $p v=p$. Then $\mathscr{A} p \subset \mathscr{A}(v)$, whence $\mathscr{A} q \subset \mathscr{A}(v)$. Since $\mathscr{B}>\mathscr{A}$, $\mathscr{B} p \cup \mathscr{B} q \subset \mathscr{A}(v)$. Thus $q=q v$ on $\mathscr{B}$.

There exists $r \in M$ with $r p=q v$. Set $\alpha=r u \in G$. Then $\alpha p=q v$ and thus $\alpha p=q$ on $\mathscr{B}$. 
Let $f \in \mathscr{A}$. Then $f \alpha p=f q=f p$. Let $p n=\omega$. Then $f \alpha=f \alpha \omega=f \alpha p n=f p n=f \omega=f$ i.e. $\alpha \in G(\mathscr{A})$.

If $p|\mathscr{B}=q| \mathscr{B}$, then the above relations are true for $(f \in \mathscr{B})$ proving that in this case $\alpha \in G(\mathscr{B})$.

24. Definition. Let $\mathscr{A}, \mathscr{B}$ be $T$-subalgebras of $\mathscr{M}$. Then $\mathscr{B}$ is a group extension of $\mathscr{A}$ if $\mathscr{B}>\mathscr{A}$ and $G(\mathscr{B})$ is a normal subgroup of $G(\mathscr{A})$.

25. Lemma. Let $\mathscr{A}, \mathscr{B}$ be $T$-subalgebras of $\mathscr{M}$ with $\mathscr{B}>\mathscr{A}$. Then $\mathscr{B}$ is a group extension of $\mathscr{A}$ if and only if $\mathscr{B} \alpha \subset \mathscr{B}(\alpha \in G(\mathscr{A}))$.

Proof. Assume $\mathscr{B}$ is a group extension of $\mathscr{A}$. Let $\alpha \in G(\mathscr{A}), \mathscr{D}$ the subalgebra of $\mathscr{C}$ generated by $\mathscr{B} \cup \mathscr{B} \alpha$.

Let $p \in M, v \in J$ with $\mathscr{A} p \subset \mathscr{A}(v)$. Since $\alpha \in G(\mathscr{A}), \mathscr{A} \alpha p=\mathscr{A} p \subset \mathscr{A}(v)$; whence $\mathscr{B} p \cup \mathscr{B} \alpha p \subset \mathscr{A}(v)$. Thus $\mathscr{D} p \subset \mathscr{A}(v)$; i.e. $\mathscr{D}>\mathscr{A}$. Consequently $\mathscr{D}>\mathscr{B}$. If $\mathscr{D} \neq \mathscr{B}$ there would exist $\beta \in G(\mathscr{B}), \beta \notin G(\mathscr{D})$. But this is impossible since $\beta \in G(\mathscr{B})$ implies that $\alpha \beta \alpha^{-1} \in G(\mathscr{B})$ whence $\beta \in G(\mathscr{B} \alpha)$ and therefore $\beta \in G(\mathscr{D})$. Consequently $\mathscr{D}=\mathscr{B}$ and $\mathscr{B} \alpha \subset \mathscr{B}$.

Now suppose $\mathscr{B} \alpha \subset \mathscr{B}(\alpha \in G(\mathscr{A}))$. Let $\beta \in G(\mathscr{B}), f \in \mathscr{B}, \alpha \in G(\mathscr{A})$. Then $f \alpha \in \mathscr{B}$ and thus $f \alpha \beta \alpha^{-1}=f \alpha \alpha^{-1}=f$; i.e. $\alpha \beta \alpha^{-1} \in G(\mathscr{B})$.

26. Proposition. Let $\mathscr{A}, \mathscr{B}$ be $T$-subalgebras of $\mathscr{M}$, let $\mathscr{B}>\mathscr{A}$ and let $\mathscr{D}$ be the subalgebra of $\mathscr{C}$ generated by $[\mathscr{B} \alpha \mid \alpha \in G(\mathscr{A})]$. Then $\mathscr{D}$ is a group extension of $\mathscr{A}$. ( $A$ fortiori $\mathscr{D}$ is a group extension of $\mathscr{B}$.)

Proof. Let $p \in \mathscr{M}, v \in J$ with $\mathscr{A} p \subset \mathscr{A}(v)$. Then $\mathscr{A} \alpha p=\mathscr{A} p \subset \mathscr{A}(v)(\alpha \in G(\mathscr{A}))$. Since $\mathscr{B}>\mathscr{A}, \mathscr{B} \alpha p \subset \mathscr{A}(v)(\alpha \in G(\mathscr{A}))$. Hence $\mathscr{D} p \subset \mathscr{A}(v)$, and therefore $\mathscr{D}>\mathscr{A}$. Clearly $\mathscr{D} \alpha \subset \mathscr{D}(\alpha \in G(\mathscr{A}))$. The proof is completed by 25 .

27. Proposition. Let $\mathscr{A}, \mathscr{B}$ be $T$-subalgebras of $\mathscr{M}$. Then $\mathscr{B}$ is a group extension of $\mathscr{A}$ if and only if there exists a group of homeomorphisms $H$ of $|\mathscr{B}|$ onto $|\mathscr{B}|$ such that $(H,|\mathscr{B}|, T)$ is a bitransformation group with $(|\mathscr{B}| / H, T)$ isomorphic to $(|\mathscr{A}|, T)$. Moreover, $H$ is isomorphic to $G(\mathscr{A}) / G(\mathscr{B})$.

Proof. Let $\mathscr{B}$ be a group extension of $\mathscr{A}$. Let $\alpha \in G(\mathscr{A}), p, q \in M$ with $p|\mathscr{B}=q| \mathscr{B}$. By $25 \mathscr{B} \alpha \subset \mathscr{B}$, whence $\alpha p|\mathscr{B}=\alpha q| \mathscr{B}$. Thus the map $p \rightarrow \alpha p$ of $M$ onto $M$ induces a map of $|\mathscr{B}|$ onto $|\mathscr{B}|$. This last is of course an automorphism of $(|\mathscr{B}|, T)$ onto $(|\mathscr{B}|, T)$.

Now let $\alpha, \beta \in G(\mathscr{A})$ with $\alpha p|\mathscr{B}=\beta p| \mathscr{B}(p \in M)$. Then by 23 there exists $\gamma \in G(\mathscr{B})$ with $\gamma \alpha=\beta$; i.e. $\beta \alpha^{-1} \in G(\mathscr{B})$. Thus $(G(\mathscr{A}) / G(\mathscr{B}),|\mathscr{B}|, T)$ is a bitransformation group.

Let $\pi:|\mathscr{B}| \rightarrow|\mathscr{A}|$ be the canonical map; i.e. $\pi(p)=p \mid \mathscr{A}(p \in|\mathscr{B}|)$. Then by 23 $\pi(p)=\pi(q)$ if and only if $\alpha p=q$ on $|\mathscr{B}|$; i.e. $\pi^{-1} \pi(p)=[\alpha p|| \mathscr{B} \mid / \alpha \in G(\mathscr{A})]$. Thus $(|\mathscr{B}| / H, T)$ and $(|\mathscr{A}|, T)$ are isomorphic.

Now let us suppose that $(H, X, T)$ is a bitransformation group such that $(X, T)$ is minimal, $X$ is compact $T_{2}$, and $X / H$ is compact $T_{2}$. 
Let $\phi: M \rightarrow X$ be a homomorphism onto, and let $\pi: X \rightarrow X / H$ be the canonical map. Then $\phi^{*}: \mathscr{C}(X) \rightarrow \mathscr{C}(M)=\mathscr{M}$ and $\pi^{*}: C(X / H) \rightarrow C(X)$ are injective. Let $\mathscr{B}=\operatorname{im} \phi^{*}, \mathscr{A}=\operatorname{im} \phi^{*} \pi^{*}$. Then $\mathscr{B} \supset \mathscr{A}$ and $(|\mathscr{B}|, T) \cong(X, T),(|\mathscr{A}|, T) \cong(X \mid H, T)$.

For $f \in \mathscr{C}(X)$ let $\bar{f}$ be that element of $\mathscr{M}$ such that $\langle\bar{f}, t\rangle=\langle f, \phi(u t)\rangle=\langle f, \phi(u) t\rangle$ $(t \in T)$. Then the above isomorphism of $(X, T)$ onto $(|\mathscr{B}|, T)$ is given by $x \rightarrow \bar{x}$ $(x \in X)$, where $\langle\bar{f} \bar{x}, t\rangle=\langle f, x t\rangle(f \in \mathscr{C}(X), t \in T)$.

Let $h \in H$. Then $f \rightarrow f h(f \in C(X))$ is a $T$-homomorphism, where $\langle f h, x\rangle=\langle f, h x\rangle$ $(x \in X)$. Thus $h$ induces a $T$-homomorphism $\bar{h}$ of $\mathscr{B}$ into $\mathscr{B}$. ( $\bar{h}$ is in fact an isomorphism onto.)

Let $p \in M, v \in J$ with $\mathscr{A} p \subset \mathscr{A}(v)$. Then $p|\mathscr{A}=p v| \mathscr{A}$. Let $x, y \in X$ with $\bar{x}=p \mid \mathscr{B}$, $\bar{y}=p v \mid \mathscr{B}$. Then $\bar{x}|\mathscr{A}=\bar{y}| \mathscr{A}$. This means that $x$ and $y$ are on the same fiber over $X / H$. Hence there exists $h \in H$ with $y=h x$; whence $\bar{y}=\bar{h} \bar{x}$. Thus $p v=\bar{h} p$ on $\mathscr{B}$; whence $h p=p v=p v v=h p v$ on $\mathscr{B}$. However, $\bar{h}$ is an automorphism of $\mathscr{B}$ onto $\mathscr{B}$; therefore $p=p v$ on $\mathscr{B}$. Then $\mathscr{B} p \subset \mathscr{A}(v)$. This proves that $\mathscr{B}>\mathscr{A}$.

Now let $\alpha \in G(\mathscr{A})$. Pick $x, y \in X$ with $\bar{x}=\alpha|\mathscr{B}, \bar{y}=\omega| \mathscr{B} . \bar{x}|\mathscr{A}=\alpha| \mathscr{A}=\omega|\mathscr{A}=\bar{y}| \mathscr{A}$. Hence $\bar{x}=\bar{h} \bar{y}$ on $\mathscr{B}$; i.e. $\alpha \mid \mathscr{B}=\bar{h}$. But $\mathscr{B} \bar{h} \subset \mathscr{B}$. Thus $\mathscr{B} \alpha \subset \mathscr{B}$ whence $\mathscr{B}$ is a group extension of $\mathscr{A}$ by 25 .

Let $\alpha \in G(\mathscr{A})$. Since $\alpha$ is a $T$-automorphism of $\mathscr{B}$ onto $\mathscr{B}$ it induces a homeomorphism $\alpha^{*}$ on $X$ which commutes with the action of $T$. Moreover, $\alpha \in G(\mathscr{A})$ implies that $\alpha^{*} x \in \pi^{-1} \pi x(x \in X)$. Choose $x \in X$. Then there exists $h \in H$ such that $\alpha^{*} x=h x$. Then $\alpha^{*}(x t)=\left(\alpha^{*} x\right) t=(h x) t=h(x t)(t \in T)$. Since $\operatorname{cls}(x T)=X, \alpha^{*}=h$. The map $\alpha \rightarrow \alpha^{*}$ is then a homomorphism of $G(\mathscr{A})$ into $H$.

Let $h \in H$. Then there exists $p \in \beta T$ with $p \mid \mathscr{B}=\bar{h}$. Since $\mathscr{B} \subset \mathscr{M}$ and $\mathscr{B} h \subset \mathscr{B}$, $p|\mathscr{B}=p u| \mathscr{B}$. Set $\alpha=p u$. Then $\alpha \in G, \alpha \mid \mathscr{B}=\bar{h}$, whence $\alpha^{*}=h$. Since $\bar{h}$ is the identity on $\mathscr{A}, \alpha \in G(\mathscr{A})$. Thus the map $\alpha \rightarrow \alpha^{*}$ is onto. It is clear that the kernel of the above map is just $G(\mathscr{B})$. The proof is completed.

Proposition 25 states that given a minimal set $(Y, T)$ with $Y$ compact $T_{2}$ then the only effective groups $H$ which can occur in bitransformation groups $(H, X, T)$ with $(X, T)$ minimal $X$ compact $T_{2}$ and $(X / H, T)$ isomorphic to $(Y, T)$ are homomorphic images of $G(\mathscr{A})$ where $\mathscr{A}$ is the algebra corresponding to $Y$. Moreover, the kernel of the aforementioned homomorphism must be a $\tau$-closed subgroup of $G(\mathscr{A})$. It is an open question whether given a $\tau$-closed normal subgroup $K$ of $G(\mathscr{A})$ there is a group extension of $(Y, T)$ with group $G(\mathscr{A}) / K$.

Let $(H, X, T)$ be a bitransformation group, let $(F, H)$ be a transformation group with phase space $F$ and phase group $H$. Then $(H, X \times F, T)$ is a bitransformation group (where $h(x, a)=\left(h x, a h^{-1}\right),(x, a) t=(x t, a)(h \in H, x \in X, a \in F$, $t \in T)$ ), and one can form the transformation group $(X \times F / H, T)$. This is essentially what occurs in the case of a general group-like extension as the following propositions indicate.

28. Proposition. Let $(H, X, T)$ be a bitransformation group with $(X, T)$ minimal such that $X$ and $X / H$ are compact Hausdorff, let $(Y, T)$ be a minimal set with compact Hausdorff phase space $Y$, let $\phi:(X, T) \rightarrow(Y, T), \psi:(Y, T) \rightarrow(X / H, T)$ be 
homomorphisms onto such that $\psi \phi=\pi$, the canonical map of $X$ onto $X / H$, let $z_{0} \in X \mid H$ and $F=\psi^{-1}\left(z_{0}\right) \subset Y$. Then there exist an action of $H$ on $F$ and a continuous bijective homomorphism $f$ of $(Y, T)$ onto $(X \times F / H, T)$.

(Note: In general $X \times F / H$ is not Hausdorff so that $f$ need not be a homeomorphism.)

Proof. Let $\mathscr{A}, \mathscr{B}, \mathscr{D}$ be $T$-subalgebras of $\mathscr{C}$ corresponding to $X / H, Y, X$ respectively. Then we may assume that $\mathscr{A} \subset \mathscr{B} \subset \mathscr{D} \subset \mathscr{M}$. By $27, \mathscr{D}$ is a group extension of $\mathscr{A}$ with $H=G(\mathscr{A}) / G(\mathscr{D})$. Again by $27, \mathscr{D}$ is a group extension of $\mathscr{B}$ with group $K=G(\mathscr{B}) / G(\mathscr{D})$. Thus we may identify $K$ with a subgroup of $H, Y$ with the space $X / K=[K x / x \in X], F$ with the set $\left[K x / x \in H x_{0}\right]$ (where $H x_{0}=z_{0}$ ), and $\phi$ and $\psi$ with the canonical maps.

Now set $(K x) h=K\left(h^{-1} x\right)\left(x \in H x_{0}, h \in H\right)$. Then $(F, H)$ is a transformation group. Let $f(K x)=\eta\left(x, K x_{0}\right)$ where $\eta: X \times F \rightarrow X \times F / H$ is the canonical map. To see that $f$ is well defined let $k \in K, x \in X$. Then $k \in H, k\left(x, K x_{0}\right)=\left(k x,\left(K x_{0}\right) k^{-1}\right)$ $=\left(k x, K\left(k x_{0}\right)\right)=\left(k x, K x_{0}\right)$; i.e. $\eta\left(k x, K x_{0}\right)=\eta\left(x, K x_{0}\right)$.

Let $f(K x)=f(K y)$ for some $x, y \in X$. Then $\eta\left(x, K x_{0}\right)=\eta\left(y, K x_{0}\right)$, whence there exists $h \in H$ with $h x=y$ and $K\left(h x_{0}\right)=K x_{0}$. Then $h x_{0} \in K x_{0}$; i.e. $h x_{0}=k x_{0}$ for some $k \in K$. Since both $h$ and $k$ commute with the elements of $T$ and $\operatorname{cls}\left(x_{0} T\right)=X$, $h=k$. Thus $h \in K$ and $K x=K y$. Hence $f$ is injective.

Let $x \in X, K a \in F$. Then there exists $h \in H$ with $h x_{0}=a$. Hence $h\left(h^{-1} x, K x_{0}\right)$ $=\left(x, K h x_{0}\right)=(x, K a)$; i.e. $\eta\left(h^{-1} x, K x_{0}\right)=\eta(x, K a)$. Thus $f\left(K h^{-1} x\right)=\eta\left(h^{-1} x, K x_{0}\right)$ $=\eta(x, K a)$. Hence $f$ is onto.

The continuity of $f$ follows from that of $g: X \rightarrow X \times F / H$ where $g(x)=f(k x)$ $(x \in X)$. The latter is continuous since it is the product of the continuous maps $x \rightarrow\left(x, K x_{0}\right)$ of $X$ into $X \times F$ and $\eta$.

The fact that $f((K x) t)=f(K x) t$ is immediate. The proof is completed.

The relevance of 28 is seen in the following proposition.

29. Proposition. Let $\mathscr{A}, \mathscr{B}$ be $T$-subalgebras of $\mathscr{H}$. Then $\mathscr{B}>\mathscr{A}$ if and only if there exists a bitransformation group $(H, X, T)$ with $(X, T)$ minimal, $X$ compact Hausdorff and homomorphisms $\phi:(X, T) \rightarrow(|\mathscr{B}|, T), \quad \psi:(X \mid H, T) \rightarrow(|\mathscr{A}|, T)$ where $\psi$ is an isomorphism and $\psi p=\pi \phi$, where $p$ and $\pi$ are the canonical maps of $x$ onto $X / H$ and $|\mathscr{B}|$ onto $|\mathscr{A}|$ respectively.

Proof. Let $\mathscr{B}>\mathscr{A}$. Set $\mathscr{D}$ equal to the $T$-subalgebra of $\mathscr{C}$ generated by $[\mathscr{B} \alpha \mid \alpha \in G(\mathscr{A})]$. Then $(G(\mathscr{A}) / G(\mathscr{D}),|\mathscr{D}|, T)$ is the required bitransformation group and $\phi$ may be taken to be the restriction map.

The converse follows from Proposition 27.

\section{REFERENCES}

1. Robert Ellis, Point transitive transformation groups, Trans. Amer. Math. Soc. 101 (1961), 384-395.

2. - A semigroup associated with a transformation group, Trans. Amer. Math. Soc. 94 (1960), 272-281. 
3. - Global sections of transformation groups, Illinois J. Math. 8 (1964), 380-394.

4. Robert Ellis and W. H. Gottschalk, Homomorphisms of transformation groups, Trans. Amer. Math. Soc. 94 (1960), 258-271.

5. H. Furstenberg, The structure of distal flows, Amer. J. Math. 85 (1963), 477-515.

6. A. W. Knapp, Distal functions, Proc. Nat. Acad. Sci., U.S.A. 52 (1964), 1409-1412.

WESLEYAN UNIVERSTTY, Middletown, CONNECTICUT

Eidgenössische Technische Hochschule, Forschungsinstitut für Mathematik, ZÜRICH, SWITZERLAND 